\title{
Nonnegative Sums of Roots of Unity
}

\author{
Morris Newman \\ Institute for Basic Standards, National Bureau of Standards, Washington, D.C. 20234
}

(July 9, 1975)

\begin{abstract}
Let $q, n$ be integers $>1$, and let $\rho_{1}, \rho_{2}, \ldots ., \rho_{n}$ be distinct $q$ th roots of unity. It is shown that $\rho_{1}^{k}+\rho_{2}^{k}+\ldots+\rho_{n}^{k} \geqslant 0$ for all integral $k_{k}$ if and only if $n$ is a divisor of $q$ and the set $\left\{\rho_{1}, \rho_{2}, \ldots, \rho_{n}\right\}$ coincides with the set $\left\{1, \zeta_{n}, \zeta_{n}^{2}, \ldots, \zeta_{n}^{n-1}\right\}$, where $\zeta_{n}=\exp (2 \pi i / n)$.
\end{abstract}

Key words. Algebraic numbers; conjugates, roots of unity.

The theorem proved in this note has its origins in problems concerning the characterization of the spectrum of a nonnegative matrix which have been studied by S. Friedland (as yet unpublished). The question answered by the theorem was posed to the author by Dr. Friedland.

THEOREM: Let $\mathrm{q}, \mathrm{n}$ be integers $>1$, and let $\rho_{1}, \rho_{2}, \ldots, \rho_{n}$ be distinct $\mathrm{qth}$ roots of unity. Then $\rho_{1}^{k}+\rho_{2}^{k}+\ldots+\rho_{n}^{k} \geqslant 0$ for all integral $\mathrm{k}$ if and only if $\mathrm{n}$ is a divisor of $\mathrm{q}$ and the set $\left\{\rho_{1}, \rho_{2}, \ldots, \rho_{n}\right\}$ coincides with the set

$$
\left\{1, \zeta_{n}, \zeta_{n}^{2}, \ldots, \zeta_{n}^{n-1}\right\}, \text { where } \zeta_{n}=\exp (2 \pi \mathrm{i} / \mathrm{n})
$$

Proof. We first assume the nonnegativity of the sums. Then after a suitable renumbering we may put $\rho_{r}=\zeta^{a_{r}}$, where $\zeta=\zeta_{q}=\exp (2 \pi i / q)$ is a primitive qth root of unity, and

$$
0 \leqslant a_{1}<a_{2}<\ldots<a_{n} \leqslant q-1 .
$$

Put

$$
\alpha_{k}=\zeta^{a_{1} k}+\zeta^{a_{2} k}+\ldots+\zeta^{a_{n} k} .
$$

Then by the hypotheses of the theorem,

$$
\alpha_{k} \geqslant 0 \quad \text { for all integral } k \text {. }
$$

It is of course sufficient to consider only those $k$ such that $0 \leqslant k \leqslant q-1$, since $\alpha_{k+q}=\alpha_{k}$. 
We have

$$
\sum_{k=0}^{q-1} \alpha_{k}=\sum_{r=1}^{n} \sum_{k=0}^{q-1} \zeta^{a} r^{k}= \begin{cases}0, & a_{1} \neq 0 \\ q, & a_{1}=0\end{cases}
$$

Thus if $a_{1} \neq 0, \alpha_{0}+\alpha_{1}+\ldots+\alpha_{q-1}=0$, which implies by (2) that $\alpha_{0}=\alpha_{1}=\ldots=\alpha_{q-1}=0$. This is not possible, since $\alpha_{0}=n$. It follows that $a_{1}=0$, and that

$$
\alpha_{0}+\alpha_{1}+\ldots+\alpha_{q-1}=q
$$

The proof will be by induction on $\Omega(q)$, the total number of prime factors of $q$. Consider first $\Omega(q)=1$, so that $q$ is prime. Since $q$ is prime, the algebraic integers $\alpha_{1}, \alpha_{2}, \ldots, \alpha_{q-1}$ form a complete set of conjugates; and since an algebraic number is zero if and only if every one of its conjugates is zero, there are just two possibilities:

$$
\begin{array}{ll}
\alpha_{k} \neq 0, & 1 \leqslant k \leqslant q-1 . \\
\alpha_{k}=0, & 1 \leqslant k \leqslant q-1 .
\end{array}
$$

Assume first that (a) holds. Then (2) implies that $\alpha_{k}>0,1 \leqslant k \leqslant q-1$. We have from (3) that

$$
\begin{gathered}
1=\left(\alpha_{0}+\alpha_{1}+\ldots+\alpha_{q-1}\right) / q \geqslant\left(\alpha_{0} \alpha_{1} \ldots \alpha_{q-1}\right)^{1 / q} \\
\alpha_{0} \alpha_{1} \ldots \alpha_{q-1} \leqslant 1 \\
\alpha_{1} \alpha_{2} \ldots \alpha_{q-1}<1
\end{gathered}
$$

since $\alpha_{0}=n<1$. It follows that $0<\alpha_{1} \alpha_{2} \ldots \alpha_{q-1}<1$, which is a contradiction, since $\alpha_{1} \alpha_{2} \ldots \alpha_{q-1}$ is the norm of the algebraic integer $\alpha_{1}$ and so a rational integer.

Now assume that (b) holds, and that $n \leqslant q-1$. Then we must have that

Thus

$$
\alpha_{k}=0, \quad 1 \leqslant k \leqslant n
$$

$$
\sum_{r=1}^{n} \zeta^{a} r^{k}=0, \quad 1 \leqslant k \leqslant n
$$

Because of (1), the matrix $S=\left(\zeta^{a} r^{k}\right), 1 \leqslant r, k \leqslant n$, must be non-singular, which contradicts (4). Thus the assumption $n \leqslant q-1$ is not possible, and so $n=q$, and the integers $a_{r}$ must satisfy $a_{r}=r-1,1 \leqslant r \leqslant n$. Thus the result follows in this case.

Now suppose the result proved for all $q$ such that $\Omega(q)<N, N \geqslant 2$, and let $q$ be any positive integer such that $\Omega(q)=N$. Let $t$ be any integer such that $1 \leqslant t \leqslant n$. We have

$$
\begin{gathered}
\alpha_{k}=\sum_{r=1}^{n} \zeta^{a} r^{k}, \\
\left.\zeta^{-a} t^{k} \alpha_{k}=\sum_{r=1}^{n} \zeta^{(a} r^{-a}\right)^{) k}, \\
\sum_{k=0}^{q-1} \zeta^{-a} t^{k} \alpha_{k}=\sum_{r=1}^{n} \sum_{k=0}^{q-1} \zeta^{(a} r^{-a} t^{\prime k}=q,
\end{gathered}
$$


sine (1) implies that $a_{r}-a_{t} \equiv 0 \bmod q$ if and only if $r=t$.

Thus

$$
\sum_{k=0}^{q-1} \zeta \quad \alpha_{k}^{-a_{t} k}=\sum_{k=0}^{q-1} a_{k}=q
$$

$$
\sum_{k=1}^{q-1}\left(1-\zeta^{-a_{t} k}\right) \alpha_{k}=0
$$

Now use the fact that $\alpha_{k}$ is real, take the complex conjugate of both sides of (5), and add the resulting equations. The result is that

$$
\sum_{k=0}^{q-1}\left(2-\zeta^{a_{t} k}-\zeta^{-a_{t} k}\right) \alpha_{k}=0
$$

Since $2-\zeta^{a_{t} k}-\zeta^{-a_{t} k} \geqslant 0$, and $\alpha_{k} \geqslant 0$ by assumption, we conclude from (6) that

$$
\left(2-\zeta^{a_{t} k}-\zeta^{-a_{t} k}\right) \alpha_{k}=0
$$

and since

$$
2-\zeta^{a_{t} k}-\zeta^{-a_{t} k}=-\zeta^{-a_{t} k}\left(1-\zeta^{a_{t} k}\right)^{2}
$$

we may also conclude that

$$
\left(1-\zeta^{a_{t}} k^{\prime}\right) \alpha_{k}=0, \quad 1 \leqslant t \leqslant n, \quad \text { all integral } k .
$$

Now the set $\{k: 1 \leqslant k \leqslant q\}$ coincides with the set $\{q l / d: d \mid q,(l, d)=1\}$, where the notation means that $d$ runs over the positive divisors of $q$ and $l$ runs over the positive integers $\leqslant d$ and relatively prime to $d$.

Suppose first that there is no value of $d>1$ such that $d$ divides $a_{t}$ for all $t$. Then (7) and the decomposition above easily imply that $\alpha_{k}=0$ for $1 \leqslant k \leqslant q-1$ (here we must use the fact that the conjugates of $\zeta_{d}$ are precisely $\zeta_{d}^{l},(l, d)=1$, so that $\alpha_{q / d}$ vanishes if and only if $\alpha_{q l / d}$ vanishes). Then it follows as before that $n=q$, and that $a_{r}=r-1,1 \leqslant r \leqslant n$. Next suppose that there is a $d>1$ such that $d$ divides $a_{t}$ for all $t$. Put $a_{t}=d b_{t}, 1 \leqslant t \leqslant n$.

Then

$$
\alpha_{k}=\sum_{r=1}^{n} \zeta_{q}^{a_{r} k}=\sum_{k=1}^{n} \zeta_{q / d}^{b_{r k}} \geqslant 0 \text { for all integral } k,
$$

and

$$
0=b_{1}<b_{2}<\ldots .<b_{n} \leqslant q / d-1 \text {. }
$$

Since $\Omega(q / d)<N$, the induction hypothesis implies that $n$ divides $q / d$, and that

$$
b_{r}=q(r-1) / n d, \quad 1 \leqslant r \leqslant n .
$$


It follows that $n$ divides $q$ and that

$$
a_{r}=d b_{r}=q(r-1) / n d, \quad 1 \leqslant r \leqslant n .
$$

Hence the result holds for any $q$ such that $\Omega(q)=N$, and the proof (in this direction) is complete.

Finally, if $n$ divides q and $a_{r}=q(r-1) / n, 1 \leqslant r \leqslant n$, then

$$
\alpha_{k}=\sum_{r=1}^{n} \zeta^{q(r-1) / n}=\sum_{r=1}^{n} \zeta_{n}^{(r-1) k}=\left\{\begin{array}{l}
n, n / k \\
0, \text { otherwise }
\end{array}\right.
$$

so that $\alpha_{k} \geqslant 0$ for all $k$.

This completes the proof of the theorem.

(Paper 80B1-430) 